
rejection after lung transplantation. Eur Respir J 2011; 37: 164-172.

6 Sutherland ER, King TS, Icitovic N, et al. A trial of clarithromycin for the treatment of suboptimally controlled asthma. J Allergy Clin Immunol 2010; 126: 747-753.

7 Brusselle GG, Vanderstichele C, Jordens P, et al. Azithromycin for prevention of exacerbations in severe asthma (AZISAST): a multicentre randomised double-blind placebo-controlled trial. Thorax 2013; 68: 322-329.

8 Global Initiative for Asthma. Global Strategy for Asthma Management and Prevention. GINA, 2010. www.ginasthma.org

9 Greening A, Ind P, Northfield M, et al. Added salmeterol versus higher-dose corticosteroid in asthma patients with symptoms on existing inhaled corticosteroid. Lancet 1994; 344: 219-224.

10 Reddel HK, Taylor DR, Bateman ED, et al. An official American Thoracic Society/European Respiratory Society statement: asthma control and exacerbations: standardizing endpoints for clinical asthma trials and clinical practice. Am J Respir Crit Care Med 2009; 180: 59-99.

11 Birring SS, Prudon B, Carr AJ, et al. Development of a symptom specific health status measure for patients with chronic cough: Leicester Cough Questionnaire (LCQ). Thorax 2003; 58: 339-343.

12 Albert RK, Connett J, Bailey WC, et al. Azithromycin for prevention of exacerbations of COPD. N Engl J Med 2011; 365: 689-698.

13 Yamaya M, Azuma A, Takizawa H, et al. Macrolide effects on the prevention of COPD exacerbations. Eur Respir J 2012; 40: 485-494.

\title{
Use of household cleaning products, exhaled nitric oxide and lung function in children
}

\author{
To the Editor:
}

The application of domestic cleaning agents increases the risk of asthma and respiratory symptoms in adults [1], in particular when products are applied in spray form [2]. Despite the associations observed in adults, the potential effects of passive exposure on children's respiratory health have not been extensively explored. Analyses of data from birth cohorts have suggested that frequent use of cleaning agents and their use in spray form increased the risk of wheezing and lower respiratory tract infections (LRTIs) during the first year of life $[3,4]$ and the risk of persistent wheezing at school age $[5,6]$. By contrast, a cross-sectional study reported protective effects of using bleach at home on the prevalence of asthma and allergic sensitisation at school age [7]. Our study investigates the effects of the use of 10 common cleaning products on exhaled nitric oxide fraction ( $\mathrm{FeNO}$ ) and on lung function (forced vital capacity (FVC) and forced expiratory volume in $1 \mathrm{~s}$ (FEV1)) during childhood in a population-based birth cohort in Menorca, Spain [8].

Recruitment was performed during pregnancy and 482 children were enrolled at birth. Written informed consent was obtained from all participants and the study was approved by a committee on ethical practice. Questionnaires on wheezing, asthma, treatment and allergies (rhinitis, eczema or hay fever) were administered by the mother repeatedly from birth until the age of 10 years. At the age of 10-13 years, FeNO (NIOX MINO; Aerocrine AB, Solna, Sweden) and forced spirometry (EasyOne; ndd Medical Technologies, Inc., Andover, MA, USA) testing was carried out. In addition, an interviewer-led questionnaire on the frequency of use of 10 different cleaning products (bleach, ammonia, polishes or waxes, acids, solvents, furniture sprays, glass cleaning sprays, degreasing sprays, air freshening sprays, and air freshening plug-in devices) was carried out. A total of 295 individuals completed the 10-year follow-up visit and the cleaning products questionnaire and performed the FeNO and/or the lung function test.

For statistical analyses, we computed a combined spray variable incorporating the four sprays (furniture, glass cleaning, degreasing and air freshening sprays) and a semiquantitative total score for cleaning product use. The means of the reported days of use per week (never $=0,<1$ day per week $=0.5,1-3$ days per week $=2$ and 4-7 days per week=5.5) for each product were summed providing a score ranging from 0 (no exposure) to 55 (exposed to all 10 products used 4-7 days per week). Multivariable linear regression models were developed to predict log-transformed FeNO concentration and non-transformed levels of FVC and FEV1. Models were adjusted for sex, age, maternal education, parental smoking indoors, asthma medication, season of respiratory test measurement, and for height and weight for lung function measurements only. The coefficients obtained from the log-transformed FeNO models were back-transformed 
TABLE 1 Weekly use of cleaning products and adjusted ${ }^{\#}$ associations with exhaled nitric oxide fraction (FeNo), forced vital capacity (FVC) and forced expiratory volume in $1 \mathrm{~s}$ (FEV1)

\begin{tabular}{|c|c|c|c|c|}
\hline Cleaning product & n $(\%)$ & $\begin{array}{c}\text { FeNO ppb } \\
\text { GM ratio }(95 \% \mathrm{Cl})\end{array}$ & $\begin{array}{c}\text { FVC mL } \\
\beta(95 \% \mathrm{Cl})\end{array}$ & $\begin{array}{l}\text { FEV1 mL } \\
\beta(95 \% \mathrm{Cl})\end{array}$ \\
\hline Bleach & $197(67)$ & $1.05(0.86$ to 1.29$)$ & $3(-94$ to 99$)$ & $44(-33$ to 120$)$ \\
\hline Polishes or waxes (for floor or furniture) & $69(24)$ & $0.86(0.68$ to 1.08$)$ & $-100(-210$ to 9$)$ & $-30(-118$ to 57$)$ \\
\hline $\begin{array}{l}\text { Acids, including decalcifiers and liquid scale } \\
\text { removers }\end{array}$ & $44(15)$ & $1.17(0.89$ to 1.55$)$ & $-77(-208$ to 54$)$ & $-78(-183$ to 26$)$ \\
\hline Furniture sprays & $104(36)$ & $1.26(1.03$ to 1.54$)$ & $27(-71$ to 125$)$ & $-49(-128$ to 29$)$ \\
\hline Glass cleaning sprays (for windows or mirrors) & $138(48)$ & $1.16(0.95$ to 1.41$)$ & $-1(-95$ to 93$)$ & $-27(-101$ to 48$)$ \\
\hline Degreasing sprays, including oven cleaning sprays & $81(28.3)$ & $1.10(0.88$ to 1.37$)$ & $-35(-141$ to 70$)$ & $-22(-106$ to 62$)$ \\
\hline Air freshening sprays & $57(19.6)$ & 1.21 (0.95 to 1.53$)$ & $-56(-170$ to 58$)$ & $-101(-191$ to -10$)$ \\
\hline Plug-in or other electric air freshening devices & $75(25.4)$ & $1.01(0.82$ to 1.25$)$ & $2(-101$ to 105$)$ & $-40(-121$ to 42$)$ \\
\hline Score of days per week of product use $\mathrm{s}^{\S}$ & $8(5.5-12)$ & $1.08(0.95$ to 1.23$)$ & $-37(-99$ to 26$)$ & $-27(-77$ to 23$)$ \\
\hline
\end{tabular}

GM: geometric mean. ${ }^{\#}$ : adjusted for sex, age, asthma medication, season of respiratory measurement, maternal education and parental smoking. FVC and FEV1 models were additionally adjusted for height and weight. ": used at least 1 day per week. ${ }^{+}$: includes furniture, glass cleaning, degreasing and air freshening sprays. ${ }^{\S}$ : the second column shows the median (interquartile range), the third to the fifth columns show the change in FeNO, FVC and FEV1 per interquartile range increase of the score (interquartile range $=6.5$ days of product use per week).

to obtain geometric mean ratios. Potential effect modification of the associations by children's lifetime wheezing, asthma or reported allergies, were evaluated by the p-value for multiplicative interaction.

The geometric mean FeNO was $13.7 \mathrm{ppb}$ and the mean lung function values were $2.9 \mathrm{~L}$ and $2.4 \mathrm{~L}$ for FVC and FEV1, respectively. The prevalence of persistent or late onset wheezing was $14 \%$, and any history of asthma or allergies from 4 to 10 years of age were reported as $9 \%$ and $36 \%$, respectively. Bleach was the most commonly used product, while sprays were used in almost three-quarters of the homes (table 1). FeNO levels were significantly higher in children exposed to sprays and furniture sprays. The use of air freshening sprays was significantly associated with lower FEV1. No statistically significant associations were observed between the FVC and any of the cleaning products.

After stratifying the analyses for asthma or allergies, the associations between the use of cleaning products, FeNO and lung function were not consistently different for the two groups of children (data not shown). Cleaning product-related health outcome changes were either higher or lower ( $p$-value for interaction $<0.1)$ in children with asthma or allergies.

Our study suggests that bystander exposure to domestic cleaning sprays may have adverse effects on schoolage children's airway inflammation by increasing exhaled nitric oxide. In addition, exposure to air freshening sprays may decrease lung function at school age. Domestic cleaning involves exposure to a large variety of chemicals including both irritant and sensitising agents that can have adverse effect on respiratory health [9]. The use of sprays for domestic cleaning may lead to a higher degree of inhalatory exposure compared with liquid products $[2,10]$.

Our results are consistent with those reported in infants and children of the younger INMA (INfancia y Medio Ambiente (Environment and Childhood) Project) cohorts, the PARIS (Pollution and Asthma Risk: an Infant Study) and ALSPAC (Avon Longitudinal Study of Parents and Children) cohorts, and with those observed in a Belgian cross-sectional study [3-7]. However, the main findings reported in these studies are mostly based on the associations with reported symptoms or diseases. Our study adds statistically significant associations with objective measurements of airway inflammation and lung function. In line with our findings, the ALSPAC studies $[5,6]$ showed positive associations between prenatal exposure to a composite score of cleaning products and persistent wheezing at 3 and 7 years of age, and lower spirometric volumes at aged 8.5 years. The INMA and PARIS studies showed an increased risk of wheezing and LRTI in infants exposed to sprays [3,4], which is consistent with our findings in FeNO at school age. Furthermore, the previous INMA study observed adverse respiratory health effects in infants exposed to air fresheners. Finally, the Belgian cross-sectional study [7] observed a protective effect of using bleach for home cleaning on reporting of asthma and allergic sensitisation in school-aged children. We did not find significant associations between the use of bleach and the respiratory outcomes monitored in this study. 
Our cross-sectional study is nested in a longitudinal birth cohort with a long follow-up that includes prospectively collected data, starting at pregnancy and continuing for $>10$ years. This prospective design provides our cross-sectional analysis with more detailed information on environmental exposures and health outcomes. In addition, our study benefits from the objective measurement of the outcomes. However, we must consider a few limitations when interpreting our results. First, the sample size was relatively small and may have limited the power of our study. Nevertheless, several associations in our study reached statistical significance. Secondly, $35 \%$ of the recruited population were excluded because information on the household use of cleaning products and/or on respiratory tests was not available. Nevertheless, the children included were not different from those excluded regarding most characteristics (sex, atopy, asthma, parental asthma and parental smoking at home). However, as anticipated, mothers of participants were more likely to have higher education. Finally, exposure to cleaning products was assessed through parental report. Unfortunately, measurements of indoor volatile organic compounds or home inspections were not performed. Thus, some parents may have over-reported the use of cleaning products or changed their cleaning behaviour according to the asthmatic or atopic status of the parents or the child. Nevertheless, sensitivity analyses suggested that asthma, wheezing, allergy or parental asthma did not modify the observed associations and associations with the objectively measured respiratory health outcomes were apparent in children without asthma or allergic disorders.

In conclusion, the domestic use of household sprays may increase children's airway inflammation and may have adverse effects on lung function. The use of cleaning products in private homes is common and many of these products are applied in a spray formulation. Therefore, our findings may have significant implications for public health. Further investigation is required to obtain a better assessment of exposure and validation of the reported exposure, as well as to identify the underlying pathophysiological mechanisms.

@ERSpublications

Use of household cleaning agents including sprays may have adverse effects on respiratory health in school-age children http://ow.ly/nSmWd

Lidia Casas ${ }^{1,2,3}$, Jan-Paul Zock ${ }^{1,2,3}$, Maties Torrent ${ }^{4}$, Raquel García-Esteban ${ }^{1,2,3}$, Esther Gracia-Lavedan ${ }^{1,2,3}$, Anne Hyvärinen ${ }^{5}$ and Jordi Sunyer ${ }^{1,2,3,6}$

${ }^{1}$ Centre for Research in Environmental Epidemiology (CREAL), Barcelona, ${ }^{2}$ Hospital del Mar Medical Research Institute (IMIM), Barcelona, ${ }^{3}$ CIBER Epidemiología y Salud Pública (CIBERESP), Madrid, ${ }^{4}$ Area de Salud de Menorca, IB-SALUT, Menorca, and ${ }^{6}$ University Pompeu Fabra, Barcelona, Spain. ${ }^{5}$ Dept Environmental Health, National Institute for Health and Welfare, Kuopio, Finland.

Correspondence: L. Casas, Centre for Research in Environmental Epidemiology, Dr Aiguader 88, 08003 Barcelona, Spain. E-mail: 1casas@creal.cat

Received: April 152013 | Accepted: May 092013

Support statement: This work was funded by Fondo de Investigacion Sanitaria, ISCIII, Ministerio de Sanidad y Servicios Sociales, Spain (Grant numbers 97/0588, 00/0021-2, G03/176, PI061756 and PS0901958), EC Contract QLK4-CT-200000263 and Fundacio Roger Torne (Barcelona, Spain). In addition, this work was supported by the European Commission as part of HITEA (Health Effects of Indoor Pollutants: integrating microbial, toxicological and epidemiological approaches), grant agreement no. 211488 under the Seventh Framework Programme, Topic ENV.2007.1.2.1.1. "Indoor air pollution in Europe: An emerging environmental health issue".

Conflict of interest: None declared.

Acknowledgements: The authors would like to acknowledge all the teachers and parents of the children from Menorca Island for patiently answering the questionnaires, all the psychologists who coordinated the fieldwork, and the nurses and administrative staff from the Primary Health Care Centre of Maó, Menorca, for administrative, technical and material support.

\section{References}

1 Medina-Ramón M, Zock JP, Kogevinas M, et al. Asthma symptoms in women employed in domestic cleaning: a community based study. Thorax 2003; 58: 950-954.

2 Zock JP, Plana E, Jarvis D, et al. The use of household cleaning sprays and adult asthma: an international longitudinal study. Am J Respir Crit Care Med 2007; 176: 735-741.

3 Casas L, Zock JP, Carsin AE, et al. The use of household cleaning products during pregnancy and lower respiratory tract infections and wheezing during early life. Int J Public Health 2012 [In press DOI: 10.1007/s00038-012-0417-2].

4 Herr M, Just J, Nikasinovic L, et al. Influence of host and environmental factors on wheezing severity in infants: findings from the PARIS birth cohort. Clin Exp Allergy 2012; 42: 275-283.

5 Sherriff A, Farrow A, Golding J, et al. Frequent use of chemical household products is associated with persistent wheezing in pre-school age children. Thorax 2005; 60: 45-49.

6 Henderson J, Sherriff A, Farrow A, et al. Household chemicals, persistent wheezing and lung function: effect modification by atopy? Eur Respir J 2008; 31: 547-554. 
7 Nickmilder M, Carbonnelle S, Bernard A. House cleaning with chlorine bleach and the risks of allergic and respiratory diseases in children. Pediatr Allergy Immunol 2007; 18: 27-35.

8 Guxens M, Ballester F, Espada M, et al. Cohort Profile: The INMA - INfancia y Medio Ambiente - (Environment and Childhood) Project. Int J Epidemiol 2012; 41: 930-940.

9 Wolkoff P, Schneider T, Kildesø J, et al. Risk in cleaning: chemical and physical exposure. Sci Total Environ 1998; 215: 135-156.

10 Bello A, Quinn MM, Perry MJ, et al. Characterization of occupational exposures to cleaning products used for common cleaning tasks - a pilot study of hospital cleaners. Environ Health 2009; 8: 11.

\title{
Concurrent coxibs and anti-platelet therapy unmasks aspirin-exacerbated respiratory disease
}

\author{
To the Editor:
}

Aspirin-exacerbated respiratory disease (AERD) is a clinical tetrad of chronic hypertrophic eosinophilic sinusitis, nasal polyps, asthma and sensitivity to any medication that inhibits cyclooxygenase (COX)-1, namely aspirin and other nonsteroidal anti-inflammatory drugs (NSAIDs) [1].

The final metabolites of the degradation of arachidonic acid via COX-1 pathway are thromboxanes, prostacyclin and prostaglandins (PG); the most crucial ones are $\mathrm{PGE}_{2}$ and $\mathrm{PGD}_{2}$. According to the classical "cyclooxygenase" hypothesis, inhibition of COX-1, but not COX-2, triggers various mechanisms leading to asthmatic and/or nasal symptoms in AERD patients. The central mechanism was regarded as the deprivation of $\mathrm{PGE}_{2}$ as a consequence of COX-1 inhibition, which would lead to an even more increased local and systemic generation of cysteinyl leukotrienes (LT). The overproduction of cysteinyl LT, due to upregulation of $\mathrm{LTC}_{4}$ synthase and/or cysteinyl LT receptors in the airways, the hallmark of the disease, occurs at baseline as well, although at a much lower degree than after aspirin/NSAIDs intake [2].

After the introduction of the selective COX-2 inhibitors, casually referred to as coxibs, several well-designed studies reported the excellent safety profile of these new NSAIDs in patients with AERD [3, 4]. Nevertheless, shortly afterwards, as the use of coxibs extended, so did the number of case reports warning the clinicians that some AERD patients may not tolerate coxibs $[5,6]$. In fact, all the position papers and updates on AERD evaluation and management recommend giving the first full dose of these drugs in the physician's office [7].

A recent study by DAHAM et al. [8] proposes a theory that might explain the underlying mechanism. These authors demonstrate that biosynthesis of $\mathrm{PGD}_{2}$ (bronchoconstrictor and pro-inflammatory mediator) in patients with asthma (of which one-third had AERD), is increased at baseline, catalysed by constitutive COX-1 only, and is not inhibited by a short 3-day treatment with celecoxib. Meanwhile, whole body formation of $\mathrm{PGE}_{2}$ (bronchodilator and anti-inflammatory) is predominantly COX-2 dependent and decreases progressively, with a reduction of $>50 \%$ as compared to baseline, during coxib treatment.

Although none of the AERD patients in this study experienced bronchoconstriction throughout the coxib treatment, COX-2 inhibition definitely had a much lesser impact on the decrease in bronchoconstrictory $\mathrm{PGD}_{2}$ than on protective $\mathrm{PGE}_{2}$, thus creating an imbalance in the airway homeostasis, which seems to be generally well tolerated by most AERD patients, except perhaps for the small subset of them who do react to selective COX-2 inhibitors in real life. This minority supposedly includes those AERD patients suffering from a more severe form of the disease [1], and/or associating other pathogenic features, such as a reduced expression of the $\mathrm{PGE}_{2}$ receptor E-prostanoid-2 on bronchial mucosal leukocytes [9], but the mechanisms underlying coxibs intolerance are not yet completely understood. Of course in real life nonselective COX inhibitors inhibit both COX-1 and -2 in a dose- and potency-dependent fashion, and other mechanisms, such as mast-cell degranulation, are involved, thus inducing reactions in all AERD patients [1,2].

We herein report on an unusual case of AERD that supports and illustrates this hypothesis. The patient is a 75-year-old male with personal history of hypertension, dyslipidaemia and a stroke in 2001, for which he has been receiving treatment with acetylsalicylic acid (ASA) $100 \mathrm{mg}$ per day. In 2006 he started to complain of perennial nasal congestion and occasional hyposmia, and has been followed up ever since by an ear, nose 